\title{
Current mode pulse width modulation/pulse position modulation based on phase lock loop
}

\author{
Pichet Wisartpong, ${ }^{*}$ Vorapong Silaphan, ${ }^{*}$ \\ Sunee Kurutach, ${ }^{*}$ Paramote Wardkein ${ }^{* *}$
}

\begin{abstract}
In this paper, the fully integrated CMOS current mode PLL with current input injects at the place of input or output of the loop filter without summing amplifier circuit. It functions as PPM and PWM circuit is present. In addition, its frequency response is an analysis which electronic tuning BPF and LPF are obtained. The proposed circuit has been designed with 0.18 $\mu \mathrm{m}$ CMOS technology. The simulation results of this circuit can be operated at $2.5 \mathrm{~V}$ supply voltage, at center frequency $100 \mathrm{MHz}$. The linear range of input current can be adjusted from $43 \mu \mathrm{A}$ to $109 \mu \mathrm{A}$, and the corresponding duty cycle of pulse width output is from $93 \%$ to $16 \%$ and the normalized pulse position is from 0.93 to 0.16 . The power dissipation of this circuit is $4.68 \mathrm{~mW}$ with the total chip area is $28 \mu \mathrm{m} \times 60 \mu \mathrm{m}$.
\end{abstract}

K e y w o r d s: pulse width modulation, pulse position modulation, phase lock loop, current mode

\section{Introduction}

Modulation is a process of varying some property of periodic signal (or carrier signal) such as amplitude, phase or frequency. In general, the carrier signal can be used cosine wave signal for analog communication systems or square wave signal for digital communication systems. For digital modulation, if the carrier wave is a square wave signal, probably called a pulse modulation, which has three properties to generate the modulating signal.

Pulse width modulation (PWM) or pulse duration modulation (PDM) is one of pulse modulation techniques, using amplitude of the analog input signal to control a width of the positive pulse or duty cycle of the pulse period. The PWM technique is frequently utilized in many applications such as switching converters or motor control in control systems or power electronics, modulation/demodulation or power amplifier in communication system etc.

The other property of pulse modulation technique uses modulating an input signal to vary the position of the positive pulse within the pulse period. In general, it can be called Pulse Position Modulation (PPM). In PPM technique, the modulated signal is transmitted with short positive pulses and all pulses have both the same positive pulse width and amplitude. The PPM signal has been utilized to improve efficiency of PWM, it fixes a width of the pulse and decreases to small as possible. The PPM technique has an advantage in power output signal is less than the PWM technique. Finally, pulse amplitude modulation (PPM) which is to be convey information with varying magnitude of positive pulse of square wave carrier proportion directly to information signals.
In general, to generate the PWM signal, we can compare between the modulating signals with a reference signal which is simple to make a circuit. However, it has a problem about the drift of the carrier frequency. Due to this problem, many researches are proposed to improve this problem by using the phase lock loop (PLL) to generate the PWM signal. Because the PLL is a well-known in a negative feedback system and gives a high stability. Thus, it is an important in many areas such as controlling motor speed, modulate/demodulate, frequency synthesizer, phase shifter, carrier recovery and etc.

Many years ago, the researchers proposed a pulse width modulation or pulse position modulation based on phase lock loop [1-9]. They classified it into 2 operation types, which consisted of operating in voltage mode [1-4] and current mode [5-7]. The advantage of current mode is better than voltage mode such as larger dynamic range, high signal bandwidth, high linearity and low power etc.

From review the current mode PLL [5] and PWM based on PLL [6-7] which proposed to apply the current mode into certain parts such as a loop filter, while other parts are operated in voltage mode such as VCO or phase detector. Therefore, in this paper proposed a fully current mode PLL with RS F/F phase detector for simultaneous PWM/PPM signals generation by using CMOS Technology. For avoiding some of the limitation or disadvantage of voltage mode such as high voltage supply etc.

The proposed model and circuit description are shown at first, next the simulation and the results of this proposed circuit by testing the characteristic of individual parts and then, combined them to full current mode PWM/PPM are presented.

Faculty of Engineering, Department of Telecommunication, Mahanakorn University of Technology, Nong Chok, Bangkok 10530, Thailand, ** Faculty of Engineering, Department of Telecommunication, King Mongkut's institute of Technology Ladkrabang, Ladkrabang, Bangkok 10520, Thailand, wpichet@mut.ac.th 


\section{The proposed Current Mode PWM/PPM and Circuit Description}

\subsection{System Description and Analysis}

In this article, pulse width and pulse position modulator by using current mode phase lock loop is proposed. It composts of current phase detector, current loop filter, and current control oscillator, which the reference signal and the output signal of current control oscillator are converted to be mono pulse signals and they are fed to be the input of RS F/F to detect the phase difference between each other. The high frequency component of the output pulse signal of $\mathrm{RS} F / \mathrm{F}$ is eliminated by using a current loop filter and then its output is used to control the output frequency of the current control oscillator. The block diagram of phase lock loop can be shown as Fig. 1

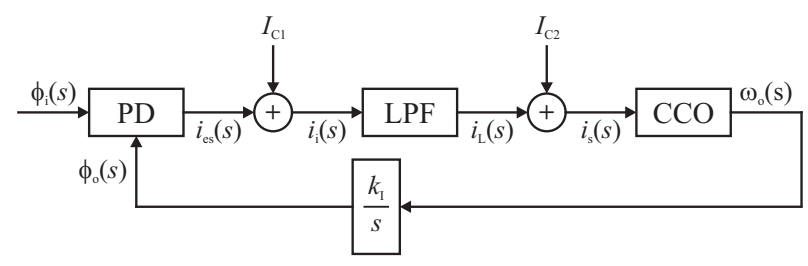

Fig. 1. Block diagram of Current Mode pwm / ppm Based on pll

From Fig. 1, the meaning of the symbolic is described by the following,

$\Phi_{i}(s)$ is a Laplace transform of an input phase function $\phi i(t)), \Phi_{0}(s)$ is a Laplace transform of an output phase function $\phi_{0}(t), \Delta \Phi(s)$ is a Laplace transform of a phase differences $\delta \phi(t), i_{L}(s)$ is a Laplace transform of an output loop filter $i_{L}(t), \Omega_{L}(s)$ is a Laplace transform of an output frequency function of CCO, $F(s)$ is a transfer function of a loop filter, $\Omega_{r}(s)$ is a Laplace transform of an output frequency function of CCO when $i_{L}(t)=0$, $k_{d}$ is a gain of a phase detector, $k_{L}$ is a gain of a loop filter, and $k_{I}$ is a gain of an integrator.

From symbolic meaning above, when we find relation all of them, which $\phi_{i}(s)$ and $\phi_{0}(s)$ relationship

$$
\begin{aligned}
& \frac{F(s) k_{d} k_{0} k_{I} \phi_{0}(s)}{s}+\phi_{0}(s)=\frac{F(s) k_{d} k_{0} k_{I} \phi_{i}(s)}{s}+ \\
& +\frac{F(s) k_{0} k_{I} I_{C 1}(s)}{s}+\frac{k_{0} k_{I} I_{C 2}(s)}{s}+\frac{k_{I} \omega_{r}}{s^{2}}
\end{aligned}
$$

To obtain a second order phase lock loop. Loop filter is assumed to be first order LPF and its transfer function is $F(s)=A /\left(1+G_{s}\right)$ when it is substituted into (1), we will derive a relation as

$$
\begin{aligned}
& \frac{A k_{d} k_{0} k_{I} \phi_{0}(s)}{s(1+G s)}+\phi_{0}(s)=\frac{A k_{d} k_{0} k_{I} \phi_{i}(s)}{s(1+G s)}+ \\
& +\frac{A k_{0} k_{I} I_{C 1}(s)}{s(1+G s)}+\frac{k_{0} k_{I} I_{C 2}(s)}{s}+\frac{k_{I} \omega_{r}}{s^{2}}
\end{aligned}
$$

where it is assumed that $K=A k_{d} k_{0} k_{I}$ and it is instituted into (2) we will get their relation in s-domain followed by inverse Laplace transform of in order to get their relation in time domain

$$
\begin{gathered}
G s^{2} \phi_{0}(s)+s \phi_{0}(s)+K \phi_{0}(s)=K \phi_{i}(s)+\frac{K}{k_{d}} I_{C 1}(s)+ \\
+\frac{K}{A k_{d}}(1+G s) I_{C 2}(s)+\frac{k_{I} \omega_{r}}{s}+k_{I} G \omega_{r}(s) \\
G \frac{d^{2} \phi_{0}(t)}{d t^{2}}+\frac{d \phi_{0}(t)}{d t}+K \phi_{0}(t)=K \phi_{i}(t)+ \\
+\frac{K}{k_{d}} I_{C 1}(t)+\frac{K}{A k_{d}}\left(I_{C 2}(t)+G \frac{d I_{C 2}(t)}{d t}\right)+ \\
+k_{I} \omega_{r} u(t)+k_{I} G \omega_{r} \delta(t)
\end{gathered}
$$

Assuming $\Phi_{i}(t)=\omega i(t)+\theta_{i}$ and input signal $I_{C 2}(t)=$ 0 when $t \geq 0 u(t)=1 ; \delta(t)=0$, therefore

$$
\begin{aligned}
& G \frac{d^{2} \phi_{0}(t)}{d t^{2}}+\frac{d \phi_{0}(t)}{d t}+K \phi_{0}(t)=K \omega_{i} t+K \phi_{i}+ \\
& +\frac{K}{k_{d}} I_{C 1}(t)+k_{I} \omega_{r}
\end{aligned}
$$

At steady state

$$
\phi_{0}(t)=\omega_{i} t+\theta_{i}+\frac{I_{C 1}(t)}{k_{0}}+\frac{\omega_{r} k_{I}}{K}-\frac{\omega_{i} k_{I}}{K}
$$

So, the phase difference between the reference signal and the output of CCO is obtained as follows

$$
\phi_{D}(t)=\phi_{i}(t)-\phi_{0}(t)=\frac{\omega_{i} k_{I}}{K}-\frac{I_{C 1}(t)}{k_{0}}-\frac{\omega_{r} k_{I}}{K}
$$

From (7), it shows that, when the phase lock loop is in the lock state, the difference phase is directly proportional to the frequency of reference input signals $\omega_{i}$. In addition, it is directly varied to current control of the current control oscillator (CCO)

$$
i_{e s}(t)=\frac{\omega_{i} k_{d}}{K}-\frac{\omega_{r} k_{d}}{K}-\frac{I_{C 1}(t)}{k_{0} K}
$$

In practice, a block diagram of PLL may be written as Fig. 2

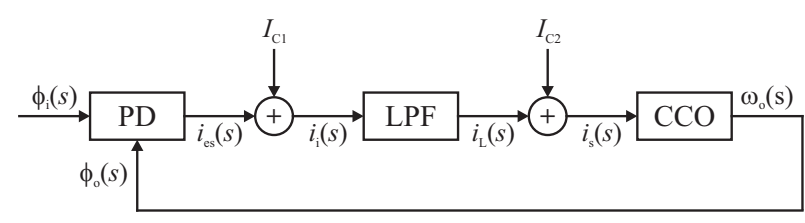

Fig. 2. Block diagram of pll in practical 

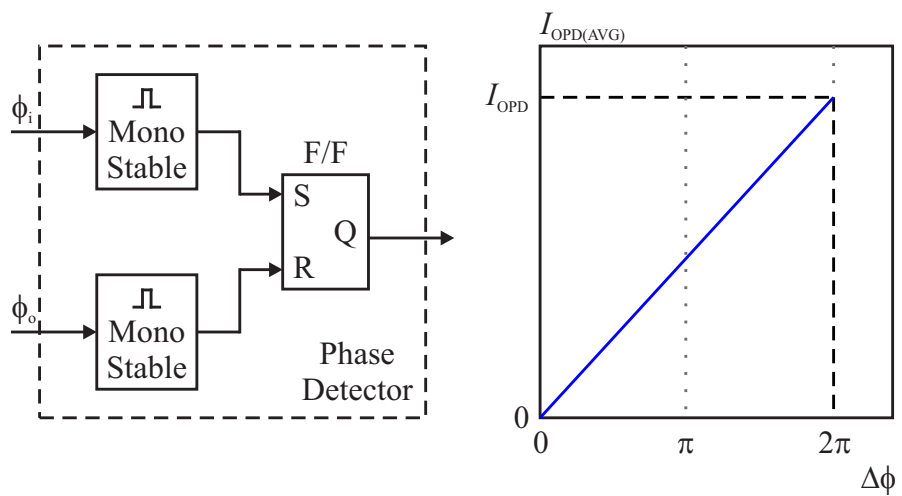

Fig. 3. Block diagram and characteristic of the RS F/F phase detector

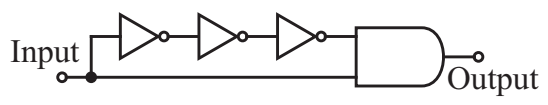

(a)

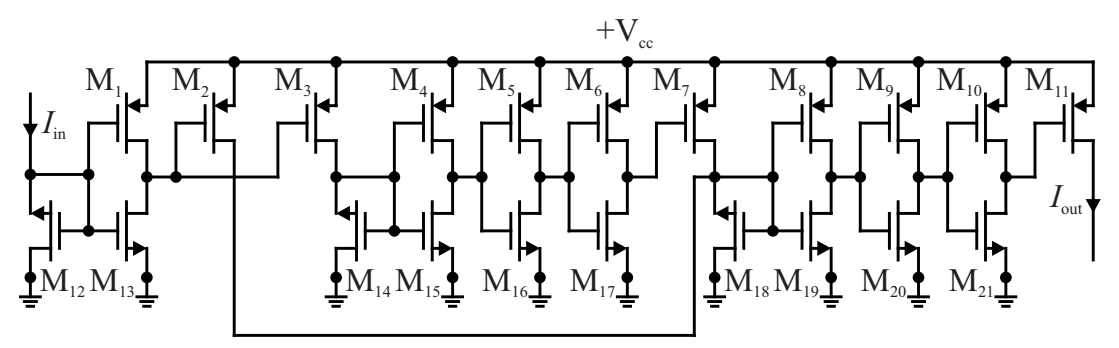

(b)

Fig. 4. Monostable Multivibrator

When $P\left(\phi_{D}(t)\right)$ is a PWM signal if $P($.$) is a phase$ function.

For frequency response from (3) under linearity property if we interested a relation between $I_{C 1}(s)$ and $i_{L}(s)$

$$
\begin{gathered}
i_{L}(s)=F(s) I_{i}(s) \\
i_{L}(s)=k_{d} F(s) \phi_{i}(s)-\frac{k_{d} k_{0} k_{I}}{s} F(s)\left(i_{L}(s)+\right. \\
\left.+I_{C 2}(s)\right)-\frac{k_{d} k_{I} \omega_{r} F(s)}{s^{2}}+I_{C 1}(s) F(s) \\
i_{L}(s)\left[1+\frac{F(s)}{s} k_{d} k_{0} k_{I}\right]=k_{d} F(s) \phi_{i}(s) \\
-\frac{k_{d} k_{0} k_{I}}{s} F(s) I_{C 2}(s)-\frac{k_{d} k_{I} \omega_{r} F(s)}{s^{2}}+I_{C 1}(s) F(s) \\
i_{L}(s) \frac{\left[G s^{2}+s+A k_{d} k_{0} k_{I}\right]}{s(1+G s)}=\frac{A k_{d} \phi_{i}(s)}{(1+G s)}- \\
\frac{A k_{d} k_{0} k_{I} I_{C 2}(s)}{s(1+G s)}-\frac{A k_{d} k_{I} \omega_{r}}{s^{2}(1+G s)}+\frac{A I_{C 1}(s)}{(1+G s)}
\end{gathered}
$$

From (12) we have relationship of one output current $\left(i_{L}(s)\right)$ and two input current $\left(I_{C 1}(s)\right.$ and $\left.I_{C 2}(s)\right)$. Thus, we obtain from two positions to feed current input.
In case 1 , we insert input current $\left(I_{C 1}(s)\right)$ between phase detector and low pass filter, and give input current $I_{C 2}=0$. We can find a transfer function between $i_{L}(s)$ with $I_{C 1}(s)$

$$
\frac{i_{L}(s)}{I_{C 1}(s)}=\frac{A s}{\left[G s^{2}+s+A k_{d} k_{0} k_{I}\right]}
$$

In case 2 , we insert input current $\left(I_{C 2}(s)\right)$ between low pass filter and $\mathrm{CCO}$, and set current input $I_{C 1}=0$. The transfer function between $i_{L}(s)$ with $I_{C 2}(s)$ is obtained

$$
\frac{i_{L}(s)}{I_{C 2}(s)}=\frac{-A k_{d} k_{0} k_{I}}{\left[G s^{2}+s+A k_{d} k_{0} k_{I}\right]}
$$

Which band pass filter and low pass filter is determined from (13) and (14) respectively.

\subsection{Phase Detector (PD)}

For the phase lock loop, the phase detector is needed to determine the phase difference between the reference signals and the output signals of current control oscillator and feedback to be input for the current control oscillator until feedback loop quiescent and approach to steady state. For phase detector circuit, it can be catalogued to two types such as digital phase detector and analog phase detector. For this here, the digital phase detector is only considered. For XOR gate PD, it achieves $0-\pi$ radian 


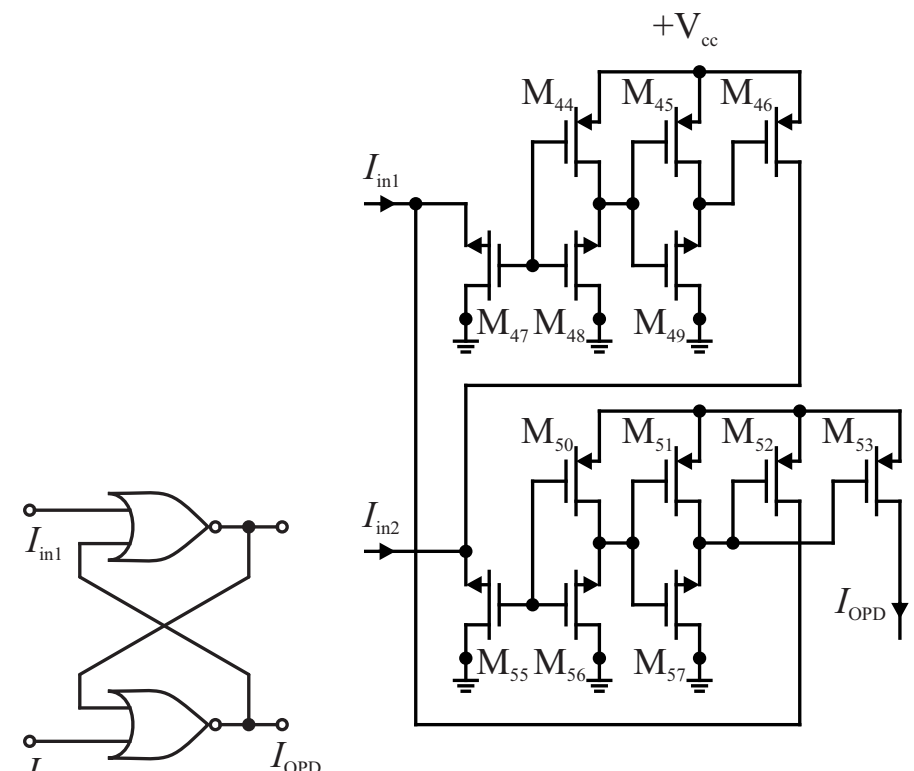

(a)

(b)

Fig. 5. R-S Flip-Flop Circuit

difference phase and its frequency output is equal a two times of reference signal. While the RS F/F PD gives 0 to $2 \pi$ radian difference phase and its frequency output is identical to the reference signal and the last one, phase/frequency detector can obtain $-2 \pi$ to $2 \pi$ radian difference phase.

To detect phase in 0 to $2 \pi$ radian range, the $\mathrm{RS} F / \mathrm{F}$ is used. Before the input signal and the reference signal are gotten for finding phase difference, the positive pulse width of both signals are adjusted with monostable multivibrators, then fed as the input of RS F/F which its block diagram is shown in Fig. 3, and this phase detector has a gain $k_{d}$

$$
k_{d}=\frac{I_{O P D}}{2 \pi}
$$

\subsubsection{Monostable Multivibrator Circuit}

In order to narrow positive pulse width, monostable multivibrators are employed; each individual composes of input current source $\left(M_{1}-M_{3}, M_{12}\right.$, and $\left.M_{13}\right)$. The input current is copied to two signals, the first one is delivered from the $M_{2}$ and the second one is delivered from $M_{3}$, which used the delay of inverter to delay the input signal, then it is fed to cascade three inverters $\left(M_{4}-M_{6}\right.$ and $\left.M_{15} M_{17}\right)$ and both signals are fed to the input of AND gate $\left(M_{7}-M_{11}\right.$ and $\left.M_{18}-M_{21}\right)$ to generate narrow positive pulse width.

\subsubsection{RS Flip-Flop}

In this paper, RS flip flop is constructed by using 2 NOR gates to connect as shown in Fig. 5(a) and Fig. 5 (b) shown the circuit diagram. The first one of NOR gate is composed of transistor $M_{44}-M_{49}$ and the second one is composed of transistor $M_{50}-M_{57}$ and delivers the output signal at drain of $M_{53}$.

\subsection{Loop filter or Low Pass Filter}

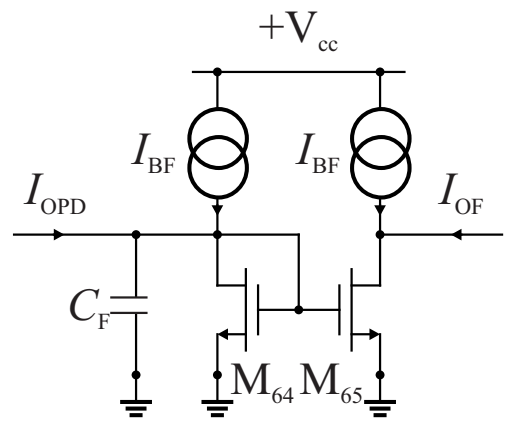

Fig. 6. Current mode first order low pass filter

The loop filter is the one of the important part of phase lock loop. It should filter a high frequency term, and pass the output signals that vary directly with phase difference, which this output signal is used to control the output frequency of the current control oscillator and feedback to the phase detector.

In this paper, the current mode first order low pass filter as shown in Fig. 6, it composes of capacitor $C_{F}$ and current mirror which its transfer function as follows

$$
F(s)=\frac{I_{O F}}{I_{O P D}}=\frac{1}{\frac{C_{F} S}{g m}+1}
$$

\subsection{Current Control Oscillator (CCO)}

Generally, the almost PLLs are constructed to operate in voltage mode. So, it uses voltage control oscillator as a one component of PLLs. Nevertheless, in this research, the PLLs operating in current mode is proposed. Therefore, it has to modify VCO to a CCO. Which relaxation CMOS oscillator voltage mode modifies the input signal 


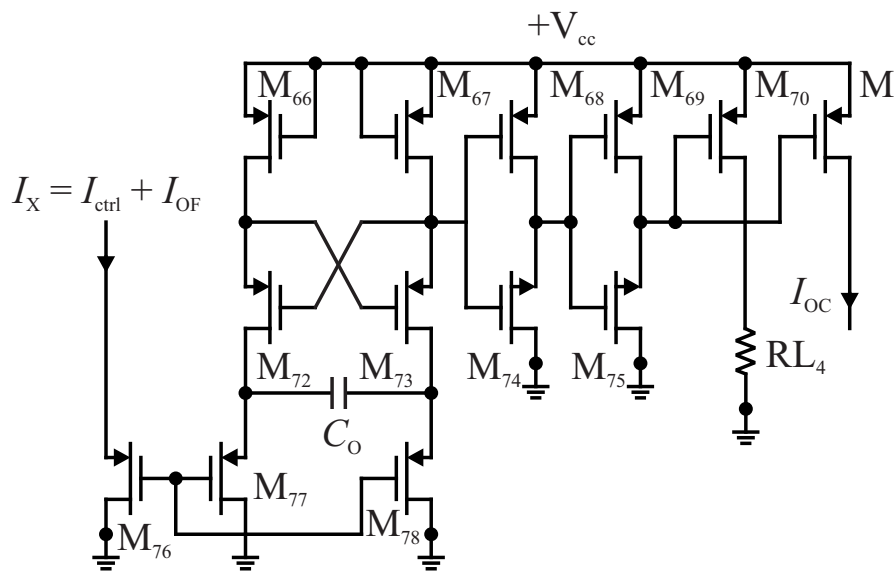

Fig. 7. Source Coupled Current Control Oscillator

as a current with current mirror, for output stage V/I converter by M71 is obtained and its circuit can be shown in Fig.6, which $I_{X}$ is a summing current between the output current of LPF $\left(I_{O F}\right)$ and external current control input $\left(I_{C t r l}\right)$. From Fig. 6, the relationship between frequency output of $\mathrm{CCO}\left(f_{O S C}\right)$ and $I_{X}$ is demonstrated as follows

$$
\begin{gathered}
f_{O S C}=\frac{1}{2 \Delta t}=\frac{I_{X} / 2}{4 C_{0} V_{T H}} \\
k_{0}=\frac{2 \pi}{4 C_{0} V_{T H}}
\end{gathered}
$$

\section{Simulation and Results}

To obtain current mode PLLs, the phase detector circuit, loop filter or low pass filter, and current control current oscillator are designed and layout chip by the Microwinds program and rule file for CMOS $0.18 \mu \mathrm{m}$ with level-3 process parameter and extract files to simulate by PSPICEs program.

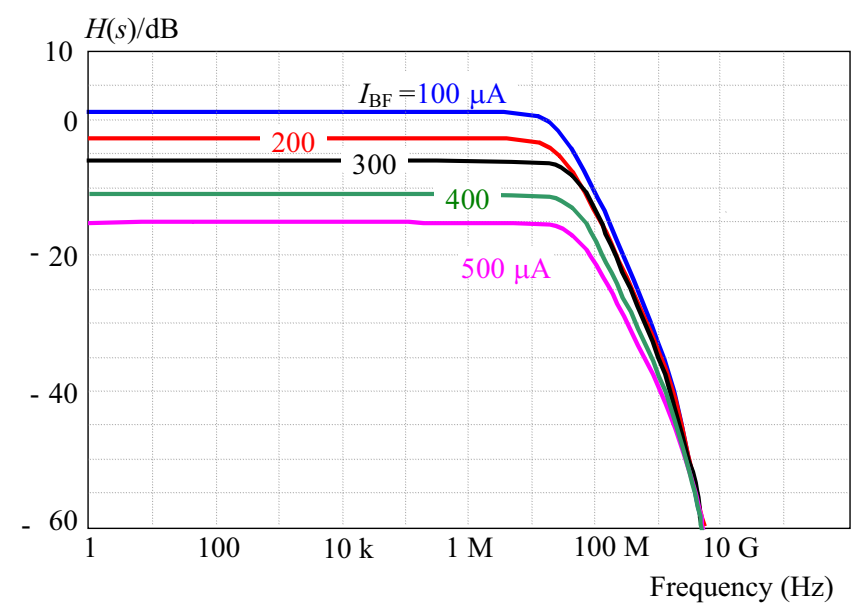

Fig. 9. Frequency response of LPF when varies $I_{B F}$

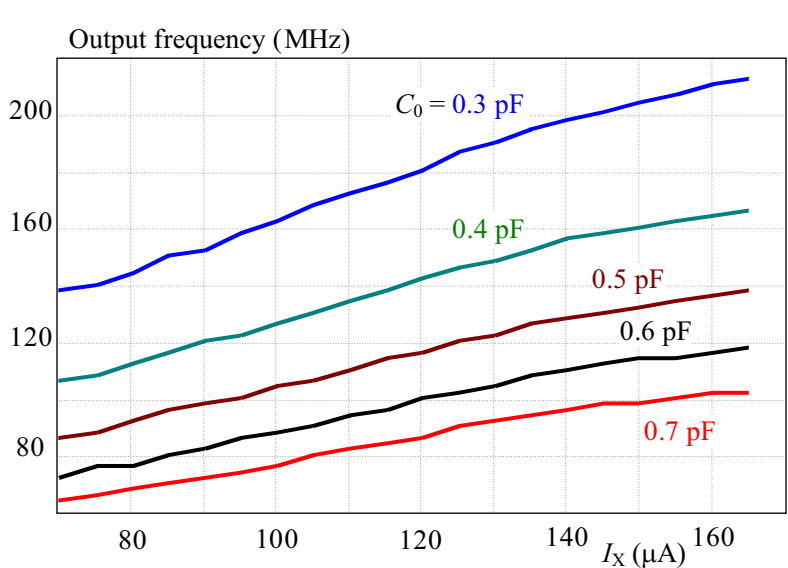

Fig. 8. Characteristic of $\mathrm{CCO}$

\begin{tabular}{|c|c|c|c|}
\hline \multirow[t]{2}{*}{ Parameter } & Typical & \multicolumn{2}{|c|}{ parameter value } \\
\hline & NMOS & PMOS & Units \\
\hline Threshold voltage (VT) & 0.35 & -0.3 & V \\
\hline Transconductance (K) & 345.31 & 172.65 & $\mu \mathrm{A} / \mathrm{V} 2$ \\
\hline
\end{tabular}

Table 1. Process parameter of CMOS $0.18 \mu \mathrm{m}$

\subsection{Simulation results of the $\mathrm{CCO}$}

For CCO simulation, the CMOS circuit as shown in Fig. 7 is simulated with supply voltage $\mathrm{VCC}=2.5 \mathrm{~V}$, adjust $C_{O}$ from $0.3 \mathrm{pF}$ to $0.7 \mathrm{pF}$ with step $0.1 \mathrm{pF}$ and $I_{X}$ is adjusted from $75 \mu \mathrm{A}$ to $170 \mu \mathrm{A}$, which $C_{O}=0.3$ $\mathrm{pF}$ is given $140 \mathrm{MHz}$ to $210 \mathrm{MHz}$ frequency range, $C_{O}$ $=0.4 \mathrm{pF}$ is given $105 \mathrm{MHz}$ to $165 \mathrm{MHz}$ frequency range, and finally, $C_{O}=0.7 \mathrm{pF}$ is given a $65 \mathrm{MHz}$ to $100 \mathrm{MHz}$ frequency range as shown in Fig. 8.

\subsection{Simulation results of loop filter}

Here, the current mode low pass filter is simulated by using the circuit in Fig. 6. Firstly, we use $C_{F}=10 \mathrm{pF}$,

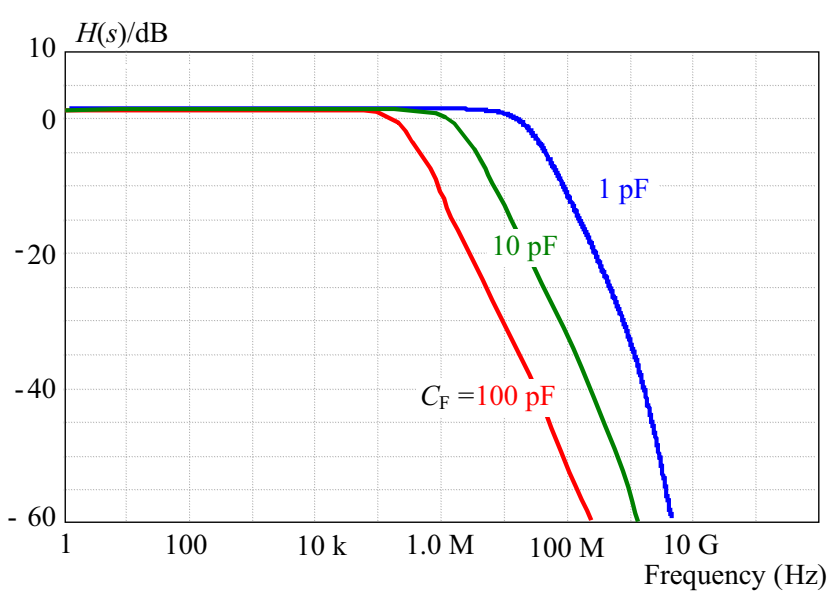

Fig. 10. Frequency response of $\mathrm{LPF}$ when varies $C_{F}$ 
Table 2. Transistor aspect ratio

\begin{tabular}{lc}
\hline Transistor & $\mathrm{W} / \mathrm{L}$ \\
& $(\mu \mathrm{m})$ \\
\hline$M_{1}, M_{5}, M_{6}, M_{9}-M_{11}, M_{13}, M_{16}, M_{17}, M_{20}-M_{22}, M_{26}, M_{27}, M_{30}-M_{33}, M_{35}, M_{38}$ & \\
$M_{39}, M_{42}-M_{45}, M_{49}-M_{51}, M_{54}, M_{56}, M_{57}, M_{59}-M_{61}, M_{63}-M_{71}, M_{74}, M_{75}$ & $0.4 / 0.2$ \\
$M_{58}, M_{72}, M_{73}$ & $0.8 / 0.2$ \\
$M_{15}, M_{18}, M_{37}, M_{40}, M_{47}, M_{55}$ & $1.0 / 1.0$ \\
$M_{4}, M_{19}, M_{25}, M_{41}$ & $0.5 / 1.0$ \\
$M_{46}, M_{52}, M_{53}$ & $0.4 / 0.4$ \\
$M_{2}, M_{3}, M_{7}, M_{12}, M_{23}, M_{24}, M_{28}, M_{34}$ & $0.4 / 1.0$ \\
$M_{8}, M_{29}, M_{77}, M_{78}$ & $1.0 / 0.5$ \\
$M_{76}$ & $2.0 / 0.5$ \\
$M_{14}, M_{36}$ & $3.0 / 3.0$ \\
$M_{62}$ & $2.0 / 2.0$ \\
\hline
\end{tabular}

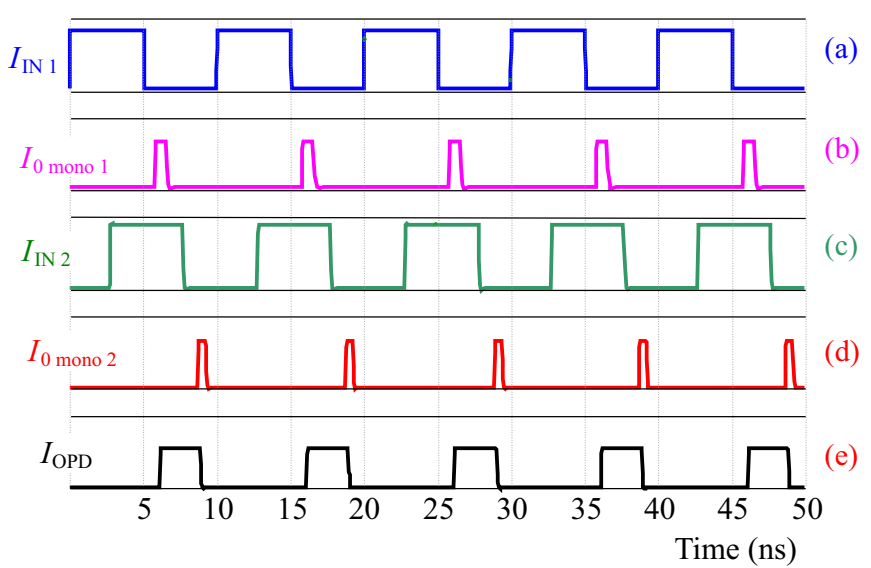

Fig. 11. Results of monostable multivibrator and pd at low phase differences

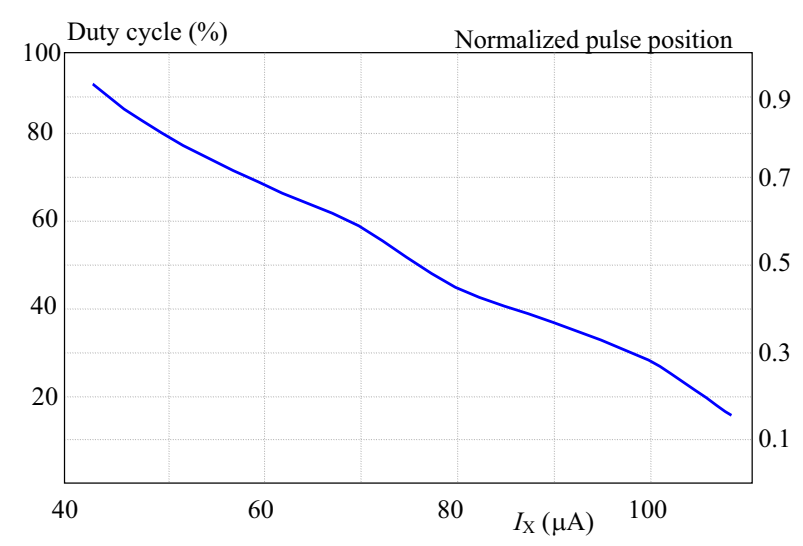

Fig. 13. DC characteristic of PWM/PPM

$V_{C C}=2.5 \mathrm{~V}$ and their current control $\left(I_{B F}\right)$ are $100 \mu \mathrm{A}$ to $500 \mu \mathrm{A}$ with step $100 \mu \mathrm{A}$, the result as shown in Fig.

9. The frequency response shown the cutoff frequency is equal to $2.46 \mathrm{MHz}, 3.37 \mathrm{MHz}, 4.06 \mathrm{MHz}, 5.7 \mathrm{MHz}$, and $7 \mathrm{MHz}$ respectively.

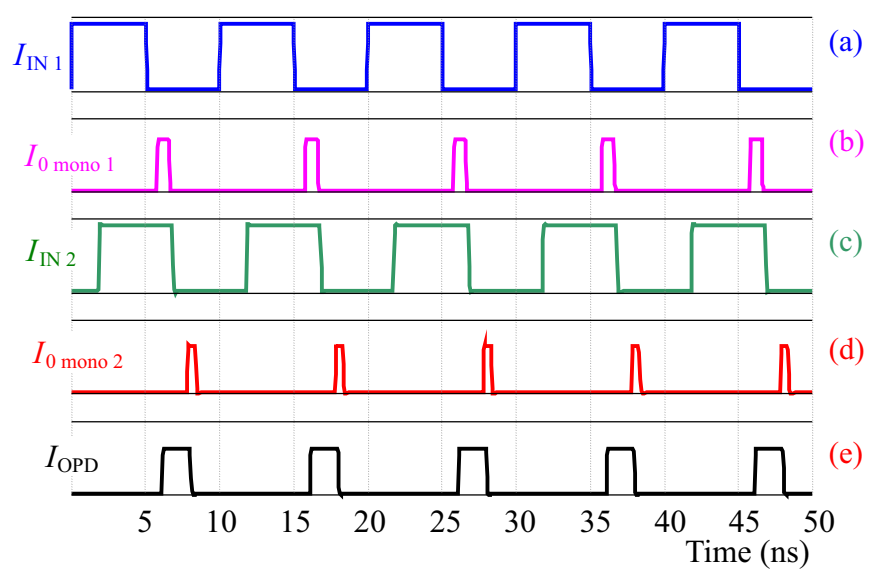

Fig. 12. Results of monostable multivibrator and pd at high phase differences

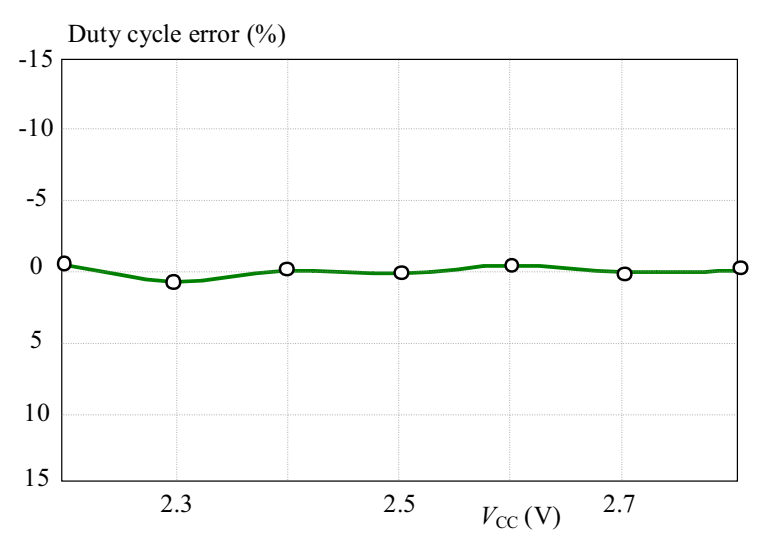

Fig. 14. Effect of power supply to duty cycle of PWM output signal

Secondly, the simulation is set by fixing control current $\left(I_{B F}\right)$ at $100 \mu \mathrm{A}$ and step change $C_{F}=1 \mathrm{pF}, 10 \mathrm{pF}$, $100 \mathrm{pF}$. The simulation result as illustrated in Fig. 10. In pass band, the current gain of them is equal to $1.24 \mathrm{~dB}$. For the cutoff frequency are $23.89 \mathrm{MHz}, 2.42 \mathrm{MHz}$, and $242.3 \mathrm{kHz}$ respectively. 


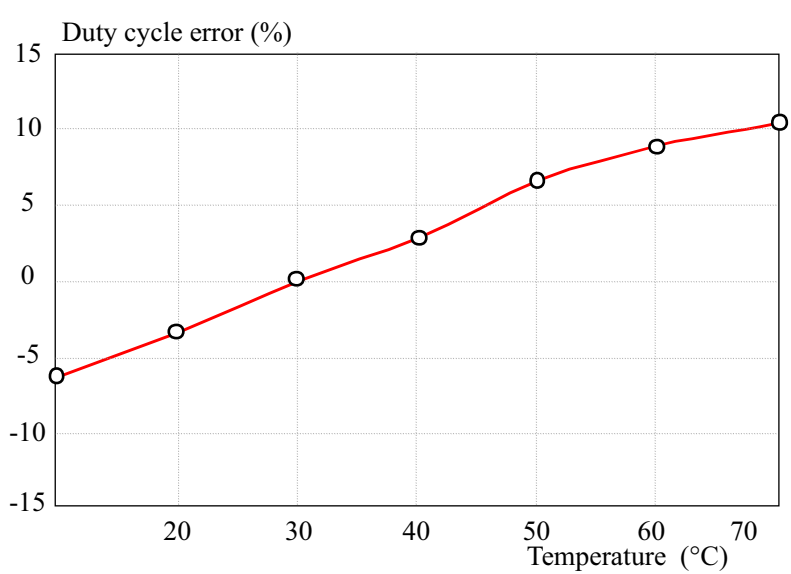

Fig. 15. Effect of temperature to duty cycle of PWM output signal

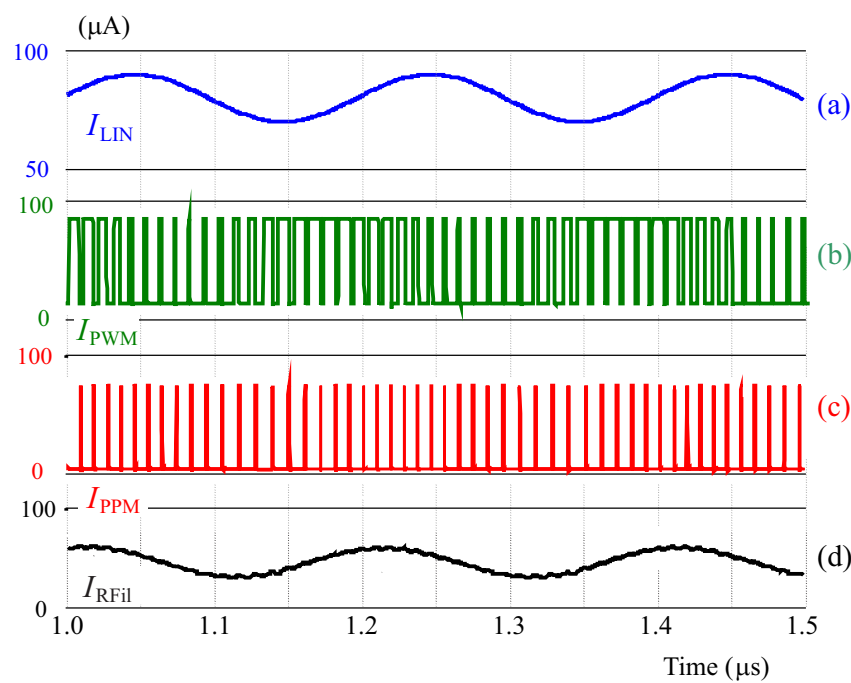

Fig. 16. Simulation results of PWM/PPM signal in time domain

\subsection{Simulation result of phase detector}

To test characteristic of the phase detector, the circuits from Fig. 4 and Fig. 5 are set up and simulated. The result is shown in Fig. 11 for low phase different and Fig. 12 for high phase different, in signal (a) and (c) are input signals of multivibrator 1 and 2, respectively, signal (b) and (d) are the output of monostable 1 and 2 , respectively, It should be noted that, monostable output is triggered with negative rise edge of the input signal and the positive pulse width is 530 ps and signal (e) is an output signal of $\mathrm{RS} \mathrm{F} / \mathrm{F}$ or PD.

\subsection{Simulation results of $P W M / P P M$}

In this section, it shows the simulation results of the PWM/PPM based on PLL system by using each part on above (PD, loop filter, $\mathrm{CCO}$ ) and combined them to PWM/PPM circuit in Fig. 13. Next, it sets all transistors by follow as Tab. 2 with DC supply voltage $\left(V_{c c}\right)=2.5$ $\mathrm{V}$, running frequency and reference frequency is $100 \mathrm{MHz}$. From the result of CCO in Fig. 8. It is assigned $\mathrm{CO}=0.6$ $\mathrm{pF}$ for $100 \mathrm{MHz}$ center frequency. In the part of signal input $\left(I_{C t r l}\right)$ is a sine wave at frequency $5 \mathrm{MHz}$. Thus, the loop filter will be assigned $C_{F}=10 \mathrm{pF}$ at $I_{B F}=100$ $\mu \mathrm{A}$ from the result of loop filter in Fig. 9 and Fig. 10.

Figure 13 shows the DC characteristic of the PWM/ PPM circuit between the input signal $\left(I_{C t r l}\right)$ in $x$-axis with a duty cycle of the pulse or pulse position on the $y$-axis. The response is a linear negative slope, which can be adjusted the duty cycle from $16 \%$ to $93 \%$ or shifted the normalized position of narrow pulse from 0.16 to 0.93 .

Figure 14 show the effect of power supply variations was also carried out. The supply voltage was varied between $2.2 \mathrm{~V}$ and $2.8 \mathrm{~V}$ in step $0.1 \mathrm{~V}$. Simulation results shown in Fig. 13 indicate that the duty cycle error of PWM output signal is between $\pm 0.6 \%$.

Figure 15 shows the Simulation of temperature effects was carried out. The temperature was swept from $10^{\circ} \mathrm{C}$ to $70{ }^{\circ} \mathrm{C}$ in steps of $10^{\circ} \mathrm{C}$. The simulation result shown in Fig. 14 the error of the duty cycle of PWM output signal is increased from $-6 \%$ to $10 \%$. It confirms that the propose circuit is sensitive to temperature variations.

Figure 16 has shown the result of PWM/PPM circuit in time domain. The trace of signals from top to bottom. The top trace is a sine wave signal input $\left(I_{C t r l}\right)$ at frequency $5 \mathrm{MHz}$, current amplitude $=28 \mu \mathrm{A}$ ppeak-topeak and offset current $=78 \mu \mathrm{A}$. The second trace is a PWM output signal it has a variation of duty cycle between $95 \%$ to $1.64 \%$. The third trace is a PPM output signal. The narrow pulse can be shifted between 9.5 to $1.64 \mathrm{~ns}$ from the position of a reference signal. The last signal is a demodulate PWM signal or signal from the output of the loop filter.

\subsection{Simulation results of frequency response of PWM/ PPM system}

From the section 2.1, we analyzed a transfer function of the propose system. It is capable to feed into 2 positions. Figure 17 illustrates the comparison results of the frequency response of this system between theory and simulation. In case 1, we feed an external signal between the output of PD and input of the loop filter. Result from (13) and simulation result shows the frequency response is a band pass filter. In case 2, we feed an external signal between the loop filter with CCO. Result from (14) and simulation result shows the frequency response is a low pass filter.

Note that another advantage of this current mode PWM/PPM based on PLL. It doesnt have an offset current input in case 1 and able to use signal input same case 2 . The difference in voltage mode, if you feed current input in case 1, you need to decrease the summary of voltage between the voltage output $P_{D}$ and voltage input signal to less than $V_{C C}$ or logic high.

The analysis frequency response shows that is a BPF or LPF for feeding input at the input and output of the loop filter, respectively, and agree with simulation results. 


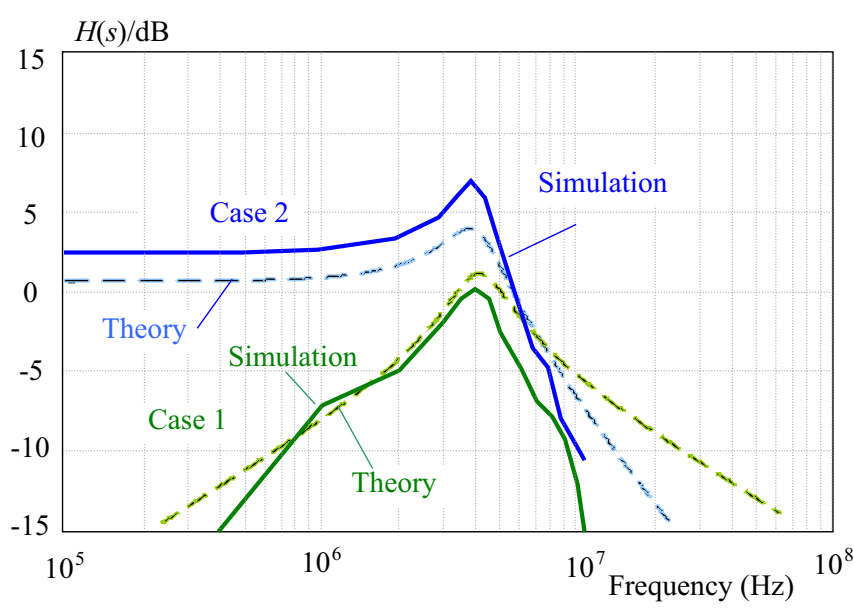

Fig. 17. Frequency response of the PWM/PPM system

\section{Conclusions}

The fully current mode PWM/PPM based on PLL have been presented in $0.18 \mu \mathrm{m}$ CMOS technology. The simulation results of current mode $\mathrm{PWM} / \mathrm{PPM}$ show that it can be operated by using a $2.5 \mathrm{~V}$ power supply. The carrier frequency or center frequency can be operated at $100 \mathrm{MHz}$ or more than if change the running frequency of CCO. The responses of the PWM signal in case of DC characteristic, it can be adjusted from $16 \%$ to $93 \%$ by varying the input control current from $93 \mu \mathrm{A}$ to $43 \mu \mathrm{A}$. In case of $\mathrm{AC}$ and $\mathrm{DC}$ characteristic, it can be adjusted duty cycle of a PWM signal from $1.64 \%$ to $95 \%$. The narrow pulse of PPM signal is equal to $530 \mathrm{ps}$. The power dissipation of this circuit is $4.68 \mathrm{~mW}$ with the total chip area is $28 \mu \times 60 \mu \mathrm{m}$. In addition, the PPM and PWM from the propose circuit is not yet depend on the supply voltage.

\section{Acknowledgements}

The author would like to thank the MICROWIND who supports the Microwind license for our layout this system.

\section{REFERENCES}

[1] M. J. Nasila, "Phase-Locked Loop Pulse Width Modulation System", US Patent US6208216B1, 2001.

[2] Y. Zheng and C. E. Saavedra, "Pulse width modulator using a phase-locked loop variable phase shifter", Proc. ISCAS2005, 2005, pp.3639-3642.

[3] P. Wisartpong, J. Koseeyaporn and P. Wardkein, "Pulse Width Modulation Based on Phase Locked Loop", Proc. ECTI-CON 2008, 2008, pp.697-700.

[4] P. Wisartpong, R. Punchalard, J. Koseeyaporn and P. Wardkein, "New Modified PLL Application Communication and Its Output Frequency Response Analysis", Far East Journal of Electronics and Communications, 2012, vol. 9, no. 1, p.25-48.
[5] D., DiClemente, and Fei and Yuan, "Current-Mode Phase Locked Loops-A New Architecture", IEEE Transaction on Circuits and Systems-II: Express Briefs, vol. 54, no. 4, pp. 303-307, April 2007.

[6] Jiann-Jong, Chen, Juing-Huei, Su, Yuh-Shyan, Hwang, Chwan -Lu, Tseng, and Hung-Yih Lin, "Wide-range high-linearity current-controlled pulse-width/delay circuit", IEICE Electronics Express, vol. 2, no. 7, pp. 217-220, April 10, 2005.

[7] Yeong-Tsair, Lin, Chi-Cheng, Wu, Jia-Long, Wu, Mei-Chu, Jen, Dong-Shiuh, Wu and Huan-Ren Cheng, "A PLL-Based Current-Mode PWM Circuit Suitable for Current-mode Control Techniques", 9th International Conference on Solid-State and Integrated-Circuit Technology (ICSICT), October 20-23, 2008, pp.2024 - 2026.

[8] Jingxue, Lu, Hyejeong, Song and Ranjit Gharpurey, "A CMOS Class-D line driver employing a phase-locked loop based PWM generator", IEEE J. Solid-State Circuits, vol. 49, no. 3, pp. 729 -739, March 2014.

[9] Kunhee, Chu and Ranjit Gharpurey, "A 40-170 MHz PLL-Based PWM Driver Using 2-/3-/5-Level Class-D PA 130 nm CMOS", IEEE J. Solid-State Circuits, vol. 51, no. 11, pp. 2639-2650, November 2016.

[10] R. J. Baker, H. W. Li and D. E. Boyce, "CMOS Circuit Design, Layout, and Simulation", IEEE Press Series on microelectronics system, 1998.

11] Arash, Ghorbannia, Delavar, Keivan, Navi and Omid Hashemi -pour, "High speed Full Swing Current Mode BiCMOS Logical Operators", IJE Transactions A: Basics, October 2007, vol. 20, no. 3 , p.211-220

Received 18 March 2017

Pichet Wisartpong was born in Thailand, in 1974. He received his BEng and MEng from the Faculty of Engineering in Mahanakorn University of Technology in 1997 and 2003, respectively. He is a lecturer in Telecommunication Engineering Department at Mahanakorn University of Technology. His research interests include analog and digital communications, communication system model.

Vorapong Silaphan was born in Thailand. He received his BInd Tech and MEng from the Faculty of Engineering in Mahanakorn University of Technology in 1993 and 2003, respectively. He is a lecturer in Telecommunication Engineering Department at Mahanakorn University of Technology. His research interests include analog and digital communications.

Sunee Kurutach was born in Thailand. She received her BEng from the Telecommunication Engineering Department at King Mongkut's institute of Technology Ladkrabang, in 1987 and her MEngSc (Communications) University of New South Wales, Australia, in 1990. Now, she is a lecturer in Telecommunication Engineering Department at Mahanakorn University of Technology. Her research interests include analog and digital communications.

Paramote Wardkein was born in Thailand. He received his MEng and DEng degrees from the Telecommunication Engineering Department at King Mongkut's institute of Technology Ladkrabang (KMITL), in 1990 and 1997, respectively. He is an Associate Professor of Telecommunication Engineering Department at KMITL. His research interests are in analog and digital communications, digital signal processing, and integrated circuit design. 